\title{
Cannabidiol in Inflammatory Bowel Diseases: A Brief Overview
}

\author{
Giuseppe Esposito, ${ }^{1 *}$ Daniele De Filippis, ${ }^{2}$ Carla Cirillo, ${ }_{3}^{3}$ Teresa Iuvone, ${ }_{1}^{2}$ Elena Capoccia, ${ }^{1}$ \\ Caterina Scuderi, ${ }^{1}$ Antonio Steardo, ${ }^{1}$ Rosario Cuomo ${ }^{3}$ and Luca Steardo ${ }^{1}$ \\ ${ }^{1}$ Department of Physiology and Pharmacology 'Vittorio Erspamer', Faculty of Pharmacy and Medicine, Sapienza University of Rome, Italy \\ ${ }^{2}$ Department of Experimental Pharmacology, Faculty of Pharmacy, Naples, Italy \\ ${ }^{3}$ Department of Clinical and Experimental Medicine, University Federico II of Naples, Naples, Italy
}

\begin{abstract}
This minireview highlights the importance of cannabidiol (CBD) as a promising drug for the therapy of inflammatory bowel diseases (IBD). Actual pharmacological treatments for IBD should be enlarged toward the search for lowtoxicityand low-cost drugs that may be given alone or in combination with the conventional anti-IBD drugs to increase their efficacy in the therapy of relapsing forms of colitis. In the past, Cannabis preparations have been considered new promising pharmacological tools in view of their anti-inflammatory role in IBD as well as other gut disturbances. However, their use in the clinical therapy has been strongly limited by their psychotropic effects. CBD is a very promising compound since it shares the typical cannabinoid beneficial effects on gut lacking any psychotropic effects. For years, its activity has been enigmatic for gastroenterologists and pharmacologists, but now it is evident that this compound may interact at extra-cannabinoid system receptor sites, such as peroxisome proliferator-activated receptor-gamma. This strategic interaction makes CBD as a potential candidate for the development of a new class of anti-IBD drugs. Copyright (C) 2012 John Wiley \& Sons, Ltd.
\end{abstract}

Keywords: cannabidiol; IBD; inflammation.

\section{THE URGENT NEED OF NEW DRUGS FOR INFLAMMATORY BOWEL DISEASES}

In the last 50 years, the incidence of inflammatory bowel diseases (IBD), namely ulcerative colitis and Crohn's disease, has dramatically increased in industrialized countries (Cosnes et al., 2011), and despite their increasing epidemiological impact, a pharmacological treatment for these diseases is still disappointingly inadequate. Prevailing therapies for IBD include chronic administration of glucocorticosteroids and sulfasalazine. Unfortunately, these drugs often possess only limited beneficial action (Fascì Spurio et al., 2012). Steroids are effective in the short-term treatment of acute flares of either form of IBD, but they are not suitable for maintenance therapies due to a variety of systemic adverse reactions (Hanauer and Stathopoulos, 1991; Engel and Neurath, 2010). Sulfasalazine and its derivative 5-aminosalicylic acid (5-ASA) are effective only in mild-to-moderate phases of the disease and in preventing relapse. Biological drugs such as monoclonal anti- tumor necrosis factor-alpha $(\mathrm{TNF} \alpha)$ antibody (Infliximab) by one side had a revolutionary impact in the management of steroid-resistant forms of IBD, obtaining encouraging results in the maintenance of the remission of the relapsing form of ulcerative colitis and Crohn's disease (Wilhelm et al., 2008). However, the long-term safety of this drug, the possibility to induce severe risks for developing cancer,

\footnotetext{
* Correspondence to: Giuseppe Esposito, M.Sc, Ph.D, Assistant professor in pharmacology and therapeutics, Department of Physiology and Pharmacology 'Vittorio Erspamer' Sapienza University of Rome P.le A. Moro 5-00185 Rome (Italy).

E-mail: giuseppe.esposito@uniroma1.it
}

i.e. leukemia and lymphoma (Rosh et al., 2007), together with the high costs of the therapy for the patients, force gastroenterologists to accurately evaluate its introduction in the therapy and to consider the risks/benefits ratio regarding its use. It is also undeniable that there is an urgent need for the identification of drugs that may efficiently control IBD evolution, with manageable side toxicity and low costs for patients. To this aim, identification and characterization of molecular targets for novel anti-IBD drugs appear to be crucial.

\section{CANNABIS PREPARATIONS: USE AND LIMITATIONS FOR IBD THERAPY}

In the USA, Cannabis preparations were indicated for the treatment of diarrhea a century ago. Nowadays, there are numerous evidences indicating the successful use of Cannabis-based products against IBD (Lahat et al., 2012). Despite the fact that Cannabis sativa has traditionally been used for centuries as an analgesic and antiinflammatory remedy, modern pharmacological therapy of inflammation with cannabinoids is still at the beginning. Cannabinoids extracted from the marijuana plant (Cannabis sativa) and synthetic cannabinoids have numerous effects on gastrointestinal (GI) functions. The natural cannabinoids comprise $\Delta^{9}$-tetrahydrocannabinol $\left(\Delta^{9}\right.$-THC), cannabichromene, cannabidiol (CBD) and cannabinol (Turner and Elsohly, 1981; Russo, 2011). The chemical and pharmacological characterization of cannabinoids and the discovery of 'endocannabinoid' compounds have been widely investigated in the last years. Pharmacological effects of natural/synthetic cannabinoids depend upon their interaction with specific 
G-protein coupled receptors (cannabinoid receptors), namely CB1 and CB2 (Grotenhermen, 2005). While CB1 receptors are mainly expressed in the central and in the peripheral nervous system (Pacher et al., 2006), CB2 receptors have been identified in the cells of immune system (Pertwee, 2006). Cannabinoid receptors, their endogenous ligands [anandamide (AEA) and 2-arachidonoyl-glycerol] together with specific enzymes [fatty acid amide hydrolase (FAAH) and monoacylglycerol-lipase] which are involved in endocannabinoid synthesis and metabolism are commonly called 'endocannabinoid system' (Pertwee, 2008).

Recent experimental data support an important role for natural cannabinoids in GI diseases (Schicho and Storr, 2012). In fact, Cannabis has been proposed to treat GI pathological conditions that range from enteric infections and inflammation to disorders of motility, emesis and abdominal pain (Izzo and Sharkey, 2010).

Converging data demonstrated that endocannabinoids may inhibit the release of a wide class of proinflammatory mediators, including interleukin-1 beta (IL-1 $\beta$ ), TNF- $\alpha$ and nitric oxide (Burstein and Zurier, 2009), thus controlling the cellular pathways leading to inflammatory responses orchestrated by massive cytokines release occurring in IBD. A huge number of studies confirmed that Cannabis preparations significantly suppress the severity of colitis in experimental animal models of IBD (Massa et al., 2004; Izzo and Camilleri, 2009). Moreover, the beneficial effect of cannabinoids has been revealed as a powerful tool to regulate gut motility and paves the way to the future development of new class of drugs capable to regulate bowel dysfunction ranging from classical ulcerative colitis and Crohn's disease to ileitis and gastric ulcers, secretory diarrhea, paralytic ileus, irritable bowel syndrome, colon cancer and gastro-esophageal reflux conditions (Izzo and Coutts, 2005).

More in detail, the ability of $\Delta^{9}$-THC to protect from inflammation, damage and motility changes in a preclinical model of IBD (Jamontt et al., 2012), as well as the ability of cannabichromene to normalize intestinal motility in the inflamed gut (Izzo et al., 2012), have been recently described. Some studies also evaluated the effect of cannabinoid compounds alone or in combination. For instance, Jamontt and colleagues not only confirmed that $\Delta^{9}$-THC and CBD exert an anti-inflammatory role in a rat model of colitis, but they also demonstrated that the combination of the two cannabinoids may be therapeutically beneficial because of a potentiated pharmacological effect (Jamontt et al., 2012).

Such evidences lead to postulate that the modulation of the activity of the cannabinoid system during gut inflammation might be a promising therapeutic tool for the treatment of those diseases characterized by inflammation. By consequence, it is not surprising that about $33-50 \%$ of people suffering from IBD have been using Cannabis to relieve IBD-related symptoms (Lal et al., 2011). This beneficial effect has been described also by Naftali et al. (2011) which reported that Cannabis may have a positive effect on Crohn's disease features, as showed by reduction in the disease activity index. In these cases, Cannabis preparations are taken as self-medication by patients with IBD in order to relieve symptoms such as abdominal pain, diarrhea and reduced appetite (GarciaPlanella et al., 2007; Lal et al., 2011).

As mentioned above, most of the anti-inflammatory pharmacological activities of cannabinoids depend upon their interaction at both $\mathrm{CB} 1$ and $\mathrm{CB} 2$ receptors in the gut (Izzo et al., 2001; Kimball et al., 2006; Storr et al., 2008; Wright et al. 2008). However, the indiscriminate binding with the receptors also in the central nervous system is accompanied to side effects such as euphoria, anxiety, psychomotor retardation and impairment of cognition and memory (Hall and Solowij, 1998). Such interaction is a self-limiting feature inherent to most of cannabinoids and explains why these compounds, despite their huge potentiality in pre-clinical studies, have had a partial and cautious clinical use (Pertwee, 2005).

\section{CANNABIDIOL IN IBD: EVIDENCE FOR ITS FAST MOVING FROM PRE-CLINICAL DATA TO CLINICAL PRACTICE}

As mentioned before, Cannabis sativa is the source of a unique set of compounds known collectively as plant cannabinoids or phytocannabinoids. Not all Cannabis compounds share at same time psychotropic and immunomodulatory functions. Among the wide range of cannabinoids extracted by this plant, one interesting exception is represented by $\mathrm{CBD}$. CBD has been isolated across the 1930s and 1940s from marijuana, but its structure and configuration were fully elucidated only in the 1960s (Mechoulam et al., 1970). (-)CBD isomer is the major non-psychotropic constituent present in Cannabis sativa. For this reason, CBD may represent the most promising candidate for clinical utilization due to its remarkable lack of any cognitive and psychoactive actions, in addition to its excellent tolerability profile in humans (Mechoulam and Hanus, 2002).

A huge amount of data produced in recent years demonstrated that CBD appears as a very promising molecule because of its anti-inflammatory, antioxidant and anti-apoptotic effects in the central nervous system (Iuvone et al., 2009). Importantly, CBD has been demonstrated to display potent anti-inflammatory and immune-modulatory properties which, together with a lack of psychotropic activity and low toxicity (Mechoulam and Hanus, 2002), make it a very promising therapeutic candidate for a variety of inflammatory and painassociated disorders, including IBD.

CBD has demonstrated the capability to mediate a strong inhibition of neutrophil chemotaxis and proliferation (Sacerdote et al., 2005), and this has been considered at the basis of its great efficacy as an anti-inflammatory drug, described both in acute and chronic animal models of inflammation (Costa et al., 2004). Along this line, in the last years, a growing number of studies highlighted the beneficial effect of CBD in gut disorders and, at the same time, they focused scientific interest toward a better comprehension of its molecular target(s) that may explain the beneficial actions in gut disturbances. Many protective functions of $\mathrm{CBD}$ have been related to the impressive antioxidant function displayed during inflammation; such antioxidant activity has been reported to markedly inhibit colon injury, inducible nitric oxide synthase expression (but not cyclooxygenase-2), and IL-1 $\beta$, IL-10 (Borrelli et al., 2009); further effects of CBD on immune cells include the inhibition of release of interferon-gamma by mononuclear cells (Formukong et al., 1988; Watzl et al., 1991) and the suppression of chemokine production by 
human B cells (Srivastava et al., 1998). All together, these observations explain why CBD has a so potent efficacy in decreasing the severity of experimental colitis in rodents (Borrelli et al., 2009; Schicho and Storr, 2012).

The great efficacy showed by CBD during colitis is partly linked to its ability to control also intestinal motility alteration. In fact, although CBD per se did not affect intestinal motility, it normalized croton oil-induced hypermotility in vivo. Similar results were obtained also in vitro, where $\mathrm{CBD}$ was able to inhibit $\mathrm{ACh}$-induced contractions in the isolated ileum from both control and croton oil-treated mice (Capasso et al., 2008). Along this line, it has been demonstrated that CBD selectively controls intestinal motor alteration in another model of hypermotility (lypopolisaccharides-induced) both in rats and in mice (Lin et al., 2011).

While it is clear why CBD is a potent anti-inflammatory drug, it appears so puzzling to understand how this molecule exerts its activity. Although it has been proposed that $\mathrm{CBD}$ may modulate endocannabinoid function through its ability to inhibit FAAH (i.e. the enzyme which hydrolyses the endocannabinoid AEA), this compound, unlike $\Delta^{9}$-THC, has very low affinity for both CB1 and CB2 receptors (Iuvone et al., 2009). As hypothesized by Capasso and colleagues, FAAH inhibition results in marked anti-inflammatory effects within the gut (Capasso et al., 2005). This is confirmed also in other gut disturbances not resembling typical IBD, such for instance intestinal sepsis (De Filippis et al., 2008). Schicho and Storr confirmed the anti-inflammatory and protective role of CBD in experimental IBD by demonstrating that the topical and systemic CBD administration improves trinitrobenzene sulfonic acid-induced colitis in mice (Schicho and Storr, 2012). Very importantly, these authors indicate that, in addition to intraperitoneal application, intrarectal delivery of CBD may represent a useful therapeutic administration route for the treatment of colonic inflammation. However, it is a common feeling that antioxidant effect cannot extensively explain per se the pharmacological capability exerted by CBD as potent inhibitor of gut inflammation.

The in-depth analysis of CBD-related pharmacology during gut disorders done by De Filippis and colleagues demonstrated that CBD may reduce intestinal inflammation severity through the control of neuro-immune axis (De Filippis et al., 2011). This study demonstrated that CBD appears as a key modulator of enteric glia-mediated neuroinflammation in the gut because of its capability to activate peroxisome proliferator-activated receptorgamma (PPAR $\gamma)$. At confirmation that CBD may exert its beneficial effects by binding PPAR $\gamma$, there are two recent studies in which the authors evaluated how CBD restores increased intestinal permeability consequent to pro-secretory agents (i.e. cytokines) application on epithelial cells (Alhamoruni et al. 2010, 2012).

CBD-mediated activation of PPAR $\gamma$ may represent a key mechanism that better explains the potent antiinflammatory action of this compound during intestinal inflammation. PPAR $\gamma$ has an inestimable value as target for novel therapeutics against IBD (Lewis et al., 2011). Activation of PPAR $\gamma$ is in fact accompanied to massive inhibition of the activity of pro-inflammatory transcription factors such as nuclear factor kappa-light-chainenhancer of activated B cells, signal transducer and activator of transcription (STAT) and activator protein-1 (Ricote et al., 1998; Esposito et al., 2011). For these reasons, CBD-mediated modulation of PPAR $\gamma$ activation may result not only a beneficial approach in the management of ulcerative colitis or Crohn's disease but also a chemopreventive pharmacological tool capable to reduce the risk of and other pathologies related to persistent inflammation of the colon, such as colon cancer. In line with these observations, a significant chemopreventive effect of CBD on experimental colon cancer in mice has been proved (Aviello et al., in press).

\section{CONCLUSION}

The beneficial and immunomodulatory effects of CBD have been widely evidenced in experimental animal models of IBD. This compound possesses an extraordinary range of beneficial effects that may slow the course of the disease, ameliorate symptoms and potentially increase the efficacy of the drugs actually available for the therapy of invalidating gut disorders such as ulcerative colitis or Crohn's disease. Because of its well-known low toxicity even in humans and its complete lack of any psychotropic unwanted effects, CBD may represent a novel molecule or a lead compound to develop new pharmacological approach to ameliorate the current therapy of IBD with fast translation from pre-clinical studies to clinical practice.

\section{Conflict of Interest}

The authors have declared that there is no conflict of interest.

\section{REFERENCES}

Alhamoruni A, Lee AC, Wright KL, Larvin M, O'Sullivan SE. 2010. Pharmacological effects of cannabinoids on the Caco-2 cell culture model of intestinal permeability. J Pharmacol Exp Ther 335: 92-102.

Alhamoruni A, Wright KL, Larvin M, O'Sullivan SE. 2012. Cannabinoids mediate opposing effects on inflammationinduced intestinal permeability. $\mathrm{Br} J$ Pharmacol 165: 2598-610.

Aviello G, Romano B, Borrelli F, et al. in press. Chemopreventive effect of the non-psychotropic phytocannabinoid cannabidiol on experimental colon cancer. $J \mathrm{Mol}$ Med [Epub ahead of print].

Borrelli F, Aviello G, Romano B, et al. 2009. Cannabidiol, a safe and non-psychotropic ingredient of the marijuana plant Cannabis sativa, is protective in a murine model of colitis. $\mathrm{J} \mathrm{Mol} \mathrm{Med} 87$ 1111-1121.
Burstein SH, Zurier RB. 2009. Cannabinoids, endocannabinoids, and related analogs in inflammation. AAPS J 11: 109-119.

Capasso R, Matias I, Lutz B, et al. 2005. Fatty acid amide hydrolase controls mouse intestinal motility in vivo. Gastroenterology 129: 941-951.

Capasso R, Borrelli F, Aviello G, et al. 2008. Cannabidiol, extracted from Cannabis sativa, selectively inhibits inflammatory hypermotility in mice. Br J Pharmaco/ 154: 1001-1008.

Cosnes J, Gower-Rousseau C, Seksik P, Cortot A. 2011. Epidemiology and natural history of inflammatory bowel diseases. Gastroenterology 140: 1785-1794.

Costa B, Colleoni M, Conti S, et al. 2004. Oral anti-inflammatory activity of cannabidiol, a non-psychoactive constituent of cannabis, in acute carrageenan-induced inflammation in the rat paw. Naunyn Schmiedebergs Arch Pharmacol 369: 294-299. 
De Filippis D, luvone T, d'amico A, et al. 2008. Effect of cannabidiol on sepsis-induced motility disturbances in mice: involvement of CB receptors and fatty acid amide hydrolase. Neurogastroenterol Motil 20: 919-927.

De Filippis D, Esposito G, Cirillo C, et al. 2011. Cannabidiol reduces intestinal inflammation through the control of neuroimmune axis. PLoS One 6: e28159.

Engel MA, Neurath MF. 2010. New pathophysiological insights and modern treatment of IBD. J Gastroentero/ 45: 571-583.

Esposito G, Scuderi C, Valenza M, et al. 2011. Cannabidiol reduces $A \beta$-induced neuroinflammation and promotes hippocampal neurogenesis through PPAR $\gamma$ involvement. PLoS One 6: e28668.

Fasci Spurio F, Aratari A, Margagnoni G, Doddato MT, Chiesara F Papi C. 2012. Oral beclomethasone dipropionate: a critical review of its use in the management of ulcerative colitis and Crohn's disease. Curr Clin Pharmacol 7: 131-136.

Formukong EA, Evans AT, Evans FJ. 1988. Analgesic and antiinflammatory activity of constituents of Cannabis sativa $L$. Inflammation 12: 361-371.

Garcia-Planella E, Marin L, Domenech E, et al. 2007. Use of complementary and alternative medicine and drug abuse in patients with inflammatory bowel disease. Med Clin (Barc) 128: 45-48.

Grotenhermen F. 2005. Cannabinoids. Curr Drug Targets CNS Neurol Disord 4: 507-530.

Hall W, Solowij N. 1998. Adverse effects of cannabis. Lancet 352 : 1611-1616.

Hanauer SB, Stathopoulos G. 1991. Risk-benefit assessment of drugs used in the treatment of inflammatory bowel disease. Drug Saf 6: 192-219.

luvone T, Esposito G, De Filippis D, Scuderi C, Steardo L. 2009. Cannabidiol: a promising drug for neurodegenerative disorders? CNS Neurosci Ther 15: 65-75.

Izzo AA, Fezza F, Capasso R, et al. 2001. Cannabinoid CB1-receptor mediated regulation of gastrointestinal motility in mice in a model of intestinal inflammation. Br J Pharmaco/ 134: 563-570.

Izzo AA, Coutts AA. 2005. Cannabinoids and the digestive tract. Handb Exp Pharmacol 168: 573-598.

Izzo AA, Camilleri M. 2009. Cannabinoids in intestinal inflammation and cancer. Pharmacol Res 60: 117-125.

Izzo AA, Sharkey KA. 2010. Cannabinoids and the gut: new developments and emerging concepts. Pharmacol Ther 126: 21-38.

Izzo AA, Capasso R, Aviello G, et al. 2012. Inhibitory effect of cannabichromene, a major non-psychotropic cannabinoid extracted from Cannabis sativa, on inflammation-induced hypermotility in mice. Br J Pharmacol 166: 1444-1460.

Jamontt JM, Molleman A, Pertwee RG, Parsons ME. 2012. The effects of Delta-tetrahydrocannabinol and cannabidiol alone and in combination on damage, inflammation and in vitro motility disturbances in rat colitis. Br J Pharmacol 160: 712-723.

Kimball ES, Schneider CR, Wallace NH, Hornby PJ. 2006. Agonists of cannabinoid receptor 1 and 2 inhibit experimental colitis induced by oil of mustard and by dextran sulfate sodium. $A m \mathrm{~J}$ Physiol Gastrointest Liver Physiol 291: G364-G371.

Lahat A, Lang A, Ben-Horin S. 2012. Impact of cannabis treatment on the quality of life, weight and clinical disease activity in inflammatory bowel disease patients: a pilot prospective study. Digestion 85: 1-8.

Lal S, Prasad N, Ryan M, et al. 2011. Cannabis use amongst patients with inflammatory bowel disease. Eur J Gastroenterol Hepatol 23: 891-896.

Lewis SN, Brannan L, Guri AJ, et al. 2011. Dietary $\alpha$-eleostearic acid ameliorates experimental inflammatory bowel disease in mice by activating peroxisome proliferator-activated receptor- $\gamma$. PLoS One 6: e24031.

Lin $\mathrm{XH}$, Yuece B, Li YY, et al. 2011. A novel CB receptor GPR55 and its ligands are involved in regulation of gut movement in rodents. Neurogastroenterol Motil 23: 862-e342.

Massa F, Marsicano G, Hermann H, et al. 2004. The endogenous cannabinoid system protects against colonic inflammation. $J$ Clin Invest 113: 1202-1209.

Mechoulam R, Shani A, Edery H, Grunfeld Y. 1970. Chemical basis of hashish activity. Science 169: 611-612.

Mechoulam R, Hanus L. 2002. Cannabidiol: an overview of some chemical and pharmacological aspects. Part I: chemical aspects. Chem Phys Lipids 121: 35-43.

Naftali T, Lev LB, Yablecovitch D, Half E, Konikoff FM. 2011. Treatment of Crohn's disease with cannabis: an observational study. Isr Med Assoc J 13: 455-458.

Pacher P, B' atkai S, Kunos G. 2006. The endocannabinoid system as an emerging target of pharmacotherapy. Pharmacol Rev 58: 389-462.

Pertwee RG. 2005. Pharmacological actions of cannabinoids. Handb Exp Pharmacol 168: 1-51.

Pertwee RG. 2006. Cannabinoid pharmacology: The first 66 years. Br J Pharmacol 147: S163-S171.

Pertwee RG. 2008. The diverse CB1 and CB2 receptor pharmacology of three plant cannabinoids: $\Delta 9$-tetrahydrocannabinol, cannabidiol and $\Delta$ 9-tetrahydrocannabivarin. $\mathrm{Br} J$ Pharmacol 153: 199-215.

Ricote M, Li A, Willson TM, Kelly C, Glass C. 1998. The peroxisome proliferator-activated receptor-gamma is a negative regulator of macrophage activation. Nature 391: 79-82.

Rosh JR, Gross T, Mamula P, Griffiths A, Hyams J. 2007. Hepatosplenic T-cell lymphoma in adolescents and young adults with Crohn's disease: a cautionary tale? Inflamm Bowel Dis 13: 1024-1230.

Russo EB. 2011. Taming THC: potential cannabis synergy and phytocannabinoid-terpenoid entourage effects. $\mathrm{Br} J$ Pharmacol 163: 1344-1364.

Sacerdote P, Martucci C, Vaccani A, et al. 2005. The nonpsychoactive component of marijuana cannabidiol modulates chemotaxis and IL-10 and IL-12 production of murine macrophages both in vivo and in vitro. J Neuroimmunol 159: 97-105.

Schicho R, Storr M. 2012. Topical and systemic cannabidiol improves trinitrobenzene sulfonic Acid colitis in mice. Pharmacology 89: 149-155.

Srivastava MD, Srivastava BI, Brouhard B. 1998. Delta9 tetrahydrocannabinol and cannabidiol alter cytokine production by human immune cells. Immunopharmacology 40: 179-185.

Storr MA, Keenan CM, Emmerdinger D, et al. 2008. Targeting endocannabinoid degradation protects against experimental colitis in mice: involvement of $\mathrm{CB} 1$ and $\mathrm{CB} 2$ receptors. $J \mathrm{Mo}$ Med 86: 925-936.

Turner CE, Elsohly MA. 1981. Biological activity of cannabichromene, its homologs and isomers. J Clin Pharmacol 21: 283S-291S.

Watzl B, Scuderi P, Watson RR. 1991. Marijuana components stimulate human peripheral blood mononuclear cell secretion of interferon-gamma and suppress interleukin-1 alpha in vitro. Int J Immunopharmacol 13: 1091-1097.

Wilhelm SM, McKenney KA, Rivait KN, Kale-Pradhan PB. 2008. A review of infliximab use in ulcerative colitis. Clin Ther 30 223-230.

Wright KL, Duncan M, Sharkey KA. 2008. Cannabinoid CB2 receptors in the gastrointestinal tract: a regulatory system in states of inflammation. Br J Pharmacol 153: 263-270. 